\title{
Proposal of a model for chemical risk preliminary assessment
}

\author{
S. Apolloni ${ }^{1}$, M. Fera ${ }^{1}$ \& R. Macchiaroli ${ }^{2}$ \\ ${ }^{1}$ Dipartimento di Progettazione e Gestione Industriale, \\ Università degli Studi di Napoli Federico II, P.le Tecchio, 80, \\ 80125 Napoli, Italy \\ ${ }^{2}$ Dipartimento di Ingegneria Aerospaziale e Meccanica, \\ Seconda Università degli Studi di Napoli, Real Casa dell'Annunziata, \\ Via Roma 29, Aversa (CE), Italy
}

\begin{abstract}
Chemical risk assessment is an increasing problem in the industrial environment due to the particular nature of the risk. The need to measure the concentration of chemical substances, moreover, may cause additional costs for assessment. So, the main problem that arises is to avoid sampling and measuring campaigns whenever possible. This objective can be achieved using a more reliable assessment model, like the one proposed in this paper. The aim of this paper is to describe the model, which is based on the assumption that the concentration of chemical agents has a log-normal distribution, and to discuss its increased reliability features.
\end{abstract}

\section{Introduction}

The study described in this paper stems from a need particularly felt in the "Health and Safety at Work" community: the need to develop reliable but easily applicable preliminary chemical risk assessment models. In other words, the proposed model is used before the application of standard assessment models proposed for example by the EN 689, EN 482 and NIOSH methods. In fact these models require, in any case, the measurement of the concentration of chemical agents. The results of the measurement campaign are then the input of a stochastic model used to assess the residual risk. All the guidelines mentioned before use a statistical approach based on the log-normal confidence test and on 
the evaluation of the mean error during the measurement. The main problem of all these methods is the cost connected to their application. In fact, most of them imply a measurement cost which represents a significant percentage of the safety total cost in a year; if one refers, in example, to middle and large plants, it may happen to find thousands of chemical products normally used during production inside the warehouse.

The aim of this work is to develop and propose a new preliminary assessment model that, on one hand, considers both the theoretical concentration of agents in the working environment and the current working practices used to minimize the risk and, on the other hand, aims at the minimization of the assessment method application cost using a calculation model that allows to avoid measurements.

\section{State of the art and open issues}

Several authors have described and designed mathematical methods to assess chemical risk.

For example in 1986 Versar proposed a mathematical tool to predict the longterm exposure to formaldehyde (Dueholm et al., [11]); actually several mathematical models have been proposed in the past for formaldehyde, all based on the assumption that its emission from a decaying source is best described by an exponential function.

Another model typically used to assess the chemical risk is the US-EPA "EXPOSURE" (Sparks, [16]). This model uses a numerical method by Levenberg-Marquardt based on a source model and a "chamber" model. The model is divided in three steps. In the modelling of emission rates the US-EPA first is used for a one-compartment analysis of the measured concentration. Secondly, an empirical two-compartment analysis was performed also using the EPA model. In this test the first step was to estimate the size of the medium-term compartment by excluding the measured concentration dominated by the initial quick decrease in concentration during the first day and also assuming that the long-term compartment model can be disregarded. The EPA one-compartment model was used to estimate a time constant and an initial source strength of the medium compartment. Next, this time constant and source strength was used by extrapolation to estimate a contribution from the medium-term compartment to each concentration measured during the first day. Finally, the short-term compartment could be described using the same model again. The third step of the procedure included a three-compartment analysis using two different procedures. The first three-compartment procedure was an exponential peeling procedure, based on the assumption that the concentration is described by

$$
E(C(t))=\sum_{i=1}^{3} a_{i} e^{-b_{i} t}
$$

where

$b_{i} \geq 0$

$\left|b_{i}\right|<\left|b_{i+1}\right|$ 
$a_{i}=b_{i}=$ parameters related to the geometrical features of the considerate environment

$E=$ the mathematical expectation operator

$t_{0,5}=\ln (2) / b_{i}$

The relation between the time constant $\left(b_{i}\right)$ and the time (half-time $t_{1 / 2}$ ) is $t_{1 / 2}=\ln (2) / b_{i}$. This is the time it takes for the concentration to reach half the initial value. The peeling procedure is initialized by an estimation of the long-term time constant, which can be determined either observing the concentration evolution or using data from the literature. Subsequently, an iterative regression procedure is used to calculate the best estimates of the short and medium term compartments using the squared residuals as an optimizing variable. The second three-compartment model uses the EXCEL-program for creation of similar estimates. No restrictions concerning the size of the long-term compartment are needed in this model. The combined result of these three types of analysis are estimates of one, two and three compartments as well as corresponding time constants from which the half-life can be calculated. Regression coefficients (squared) were used for evaluation of the curve-fitting procedure and estimates were made of the accuracy of the exposure estimates.

Some authors (Dueholm at al., [11]) suggest combining these models to introduce a more reliable model for the chemical risk assessment, and apply this model to the formaldehyde exposure.

Other authors underline the possibility to investigate workers exposure using a statistical approach based on UCL (יper Confidence Level), generally used for the low-dose risk. However this approach generally is too conservative, and is very expensive in terms of time and difficulty of calculation, especially when it's necessary to investigate a mixture of chemical agents (Hwang and Chen, 1999).

In fact some authors (Gaylor and Chen, [17]) introduced a simpler model based on the UCL method, that is based on the sum of the individual risks. But in 1999 Hwang and Chen demonstrated that this approach is a good approach for the symmetric concentration distributions but not for asymmetric ones.

Other authors (Scheringer et al., [1]) suggest to use a technique named "Scenario Based isisk $\underline{\text { Assessment" }}$ (SceBRA). This method consists of three steps: (i) analysis of the chemical product life cycle, (ii) definition of the exposure scenarios and (iii) calculation of the potential risk. Many assessments on very large systems are based on this approach, like territorial chemical risk assessment (Rood et al., [3]).

As just described, the chemical risk assessment models and methods are many and very different between them. But all of them require the execution of difficult calculations, while each of them is well suited for specific applications. There were some attempts, in the past, to create a tool for chemical risk assessment that could integrate all existing assessment models and provide a conservative risk evaluation index (Ignatowski and Rosenthal, [2]); this experiment was the "Thesaurus" project, that failed in 2005.

So, after this discussion, it is quite evident that nowadays a reliable assessment model with a simple calculation scheme is not available. This 
problem is particularly felt by small and medium enterprises (SME). For SMEs it's very important to minimize the assessment cost and, accordingly, to reduce the number of chemical agents whose concentration needs to be investigated with the methods of EN 689:1995 or NIOSH.

\section{The proposed model}

Standing the open issues described in the previous section, the aim of this work is to propose a new, simplified, preliminary assessment model.

In particular, our aim was to create an assessment model useful for small and medium enterprises and able to offer a reliable decision criteria on which chemical agents deserve deeper investigation with the support of the measurements. The research for this feature was due to the need to reduce the impact of measurement cost on the total cost of safety.

The model development starts from the following observation: usually SMEs do not (or are not able to) use and integrate available data - daily quantities used

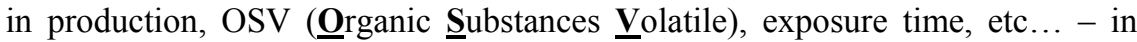
order to estimate the chemical agents concentration in the working environment. The only mean to assess chemical risk is to measure the concentration, thus incurring into a significant evaluation cost.

Coherently with the suggested goal - avoid, as far as possible, measurement campaigns - our proposal introduces an equation that allows one to compute the expected concentration of a given chemical substance based on:

$$
\text { net quantity stocked during the year of a specific chemical agent, }
$$
quantity released in the air during working time, hours worked during a year, volume of the working environment.

The equation is:

$$
C=\frac{Q \cdot \% s}{d \cdot h \cdot V}
$$

where

$C=$ concentration of the chemical agent $\left[\mathrm{g} / \mathrm{m}^{3} \mathrm{~h}\right]$

$Q=$ net yearly quantity used in production

$\% s=$ dispersion in the working environment [\#]

$d=$ yearly working days [day/year]

$h=$ daily working hours [h/day]

$V=$ volume of the working environment $\left[\mathrm{m}^{3}\right]$

All data necessary to apply the equation are usually available in all structured enterprises. In fact, the net quantity $Q$ is the difference among the quantity bought during the year and the stocked quantity at the end of the year. The 
dispersion percentage in the working environment can be inferred from safety sheets of the chemical product, in case of liquid substances, while, in case of powder products, its consistence is about 3\%-4\% in normal condition of operation, i.e., with standard ventilation conditions. The yearly working days are available for all workers, as also available is the number of hours worked by workers in presence of the substance. Finally, the volume of the working environment can be easily calculated using main geometric and architectural data of the working environment.

The importance to calculate the concentration using this formula is related also to the possibility for the enterprise auditor to compare such theoretical concentration directly with the TLV (Threshold Limit Value), generally reported on safety sheets of chemical products. The approach, therefore, has a straightforward "physical" meaning, that allows the auditor to give an immediate evaluation of the chemical risk.

The proposed model, moreover, then modifies the pure calculated concentration with a correction factor that considers all organizational and technological conditions that may have an influence on work safety conditions with a special reference to chemical risk.

This factor is composed by different terms directly related to:

$$
\text { working methods, }
$$

$$
\text { intrinsic danger of the chemical substance, }
$$$$
\text { reactivity of the chemical substance and }
$$

$$
\text { personnel awareness. }
$$

In particular, a study to identify the weights of each term was conducted, using typical methods of knowledge management. The opinions to decide the weights of each term were collected between senior safety auditors and chemical risk experts.

The first form of the correction factor $K$ is:

$$
K=\left(w_{1} \cdot T W+w_{2} \cdot D+w_{3} \cdot R+w_{4} \cdot A\right)
$$

where

$K=$ correction factor for the concentration of the chemical agent;

$w_{i}=$ weights of each term $-\mathrm{i}=1, \ldots, 4$;

$W M=$ score related to safety of working methods;

$D=$ score related to the intrinsic danger of the chemical agent;

$R=$ score related to the reactivity of the chemical agent;

$A=$ score related to the level of awareness about the chemical risk of the personnel.

Score values were determined using opinions collected among senior auditors and safety experts. The values of the single weights are shown in table 1. 
Table 1: $\quad$ Weights of individual terms in relation (3).

\begin{tabular}{|c|c|c|c|c|c|}
\hline & $W_{1}$ & $w_{2}$ & $w_{3}$ & $w_{4}$ & $\sum_{i} w_{i}$ \\
\hline Value & 0.6 & 0.2 & 0.1 & 0.1 & 1 \\
\hline
\end{tabular}

For the decision about the score to assign to working methods $(W M)$, as existing in the plant, it's possible to recognize three sub-terms, $(a)$ individual safety protections (ISP), (b) safety integrity level (SIL) of equipment and machinery and (c) type of work (TW).

For the individual safety protections assessment, the judgement is up to the auditor and the score can assume four values, (vg) very good, (g) good, (s) sufficient, (1) low.

For the safety integrity level (SIL) of equipment and machinery, it's possible to refer to the international norms IEC 61508-5-6 where the SIL levels are defined according to the presence and failure probability of control and monitoring equipment.

As far as type of work is concerned, we can define four types of work, i.e. (i) manual work, i.e., a work performed only with the help of hands or with some simple tools, (ii) semi-automated work, i.e., a work performed with the combination of man and machinery, (iii) automated work, i.e., a work performed with a limited help from human resources and (iv) fully automated or robotized work, i.e., a work that does not need men to be executed.

It's important to note that usually the individual terms are not independent: in particular, since working methods have an influence on individual safety protections and chosen SIL levels, this circumstance has to be conveniently modelled in the expression that allows to compute $W M$ as a function of the three mentioned sub-terms. The proposed sub-term combination is the following, where the relation among the first and the second term is weaker than the ones with the third:

$$
W M=(I S P+S I L) \cdot T W
$$

Note that when SIL is not applicable, the term $b$ simply equals zero.

So, the score $W M$ can be computed using the following table 2, and adding the two results from ISP and SIL.

Table 2: $\quad$ Votes for the type of works, individual safety protection and safety integrity level.

\begin{tabular}{|c|c|c|c|c|c|c|c|c|c|}
\hline $\boldsymbol{W M}$ & & \multicolumn{4}{|c|}{$I S P$} & \multicolumn{4}{|c|}{ SIL } \\
\hline & $T W$ & $(\mathrm{vg})$ & $(\mathrm{g})$ & $(\mathrm{s})$ & $(\mathrm{l})$ & 1 & 2 & 3 & 4 \\
\hline (i) & 0.7 & 0.7 & 0.8 & 0.9 & 1 & 1 & 0.9 & 0.8 & 0.7 \\
\hline (ii) & 0.6 & 0.8 & 0.9 & 1 & 1.1 & 1.1 & 1 & 0.9 & 0.8 \\
\hline (iii) & 0.5 & 0.9 & 1 & 1.1 & 1.2 & 1.2 & 1.1 & 1 & 0.9 \\
\hline (iv) & 0.4 & 1 & 1.1 & 1.2 & 1.3 & 1.3 & 1.2 & 1.1 & 1 \\
\hline
\end{tabular}


The calculation procedure can be initialized with the choice of the sub term $T W$, after it's possible to choose the sub-terms ISP and SIL and then add them to obtain the value for $W M$.

For the score to assign to the intrinsic danger of chemical agents $(D)$, with the help of the known techniques of knowledge management, it was possible to define the table of judgement (Table 3).

When a chemical substance has more than one characteristic of intrinsic danger, it's possible to combine them with the following equation:

$$
D=\max _{i} d_{i}+\frac{1}{10} \cdot \log _{e}\left[10 \cdot \sum_{k=0,13}\left(d_{k}+\frac{1}{10}\right)\right]
$$

The logarithm was selected in order to give more importance to the main intrinsic danger.

For the term $R$ the judgement can assume two values, depending upon the presence or absence of chemical agents or products reactivity features; as an example, one may refer to caustic soda, that reacts with violence when mixed with water. The values of the judgements are reported in the Table 4 .

The term $A$, according to the experts opinion, can assume three values, corresponding to different levels of awareness (Table 5).

Table 3: $\quad$ Votes for the intrinsic danger.

\begin{tabular}{|c|c|}
\hline Intrinsic Danger & $D$ \\
\hline Theratogen & 0,50 \\
\hline Mutagen & 0,50 \\
\hline Dangerous for the Environment & 0,50 \\
\hline Carcinogenic & 0,45 \\
\hline Very Toxic & 0,45 \\
\hline Toxic & 0,40 \\
\hline Corrosive & 0,40 \\
\hline Very Flammable & 0,40 \\
\hline Oxidant & 0,40 \\
\hline Dangerous for the Aquatic & 0,40 \\
\hline Organisms & 0,35 \\
\hline Noxious & 0,35 \\
\hline Flammable & 0,25 \\
\hline Irritants & \\
\hline
\end{tabular}


Table 4: $\quad$ Votes for the substance reactivity.

\begin{tabular}{|c|c|}
\hline Reactivity & $R$ \\
\hline Presence & 0.1 \\
\hline Absence & 0 \\
\hline
\end{tabular}

Table 5: $\quad$ Votes for the workers' awareness.

\begin{tabular}{|c|c|}
\hline Awareness & $A$ \\
\hline $\begin{array}{c}\text { Specific on the single } \\
\text { chemical agent risk }\end{array}$ & -0.1 \\
\hline $\begin{array}{c}\text { General on the chemical } \\
\text { risk }\end{array}$ & -0.1 \\
\hline Absent & +0.2 \\
\hline
\end{tabular}

Once the values of all terms are determined it is possible to compute the corrected value of concentration (Ccor) as

$$
C_{c o r}=K \cdot C=\left(w_{1} \cdot T W+w_{2} \cdot D+w_{3} \cdot R+w_{4} \cdot A\right) \cdot C
$$

During the construction of this model we assumed, like several authors suggest, that the statistical distribution of the measurements has a log-normal distribution (El-Baz and Nayak [10] - Tarter and Hill [8]).

Thus, our assumption is that $C_{c o r}$ is the mean of the concentration distribution, modelled as a log-normal.

The next step of the assessment model is to estimate the probability that the actual concentration in the working environment exceeds the TLV. To this aim, we must make an assumption upon what $C_{c o r}$ represents, either the geometric or the simple mean. This discussion is reported at the end of this section. The model, after having computed such probability, can give an estimate of the acceptable level of risk, e.g. the risk is assumed to be acceptable if the probability does not exceed $10 \%$, as suggested by ACGIH.

To compute such probability, assuming the concentration distribution is lognormal, it is possible to use standard log-normal tables. The use of these tables requires as an input the values of geometrical mean $(G M)$ and the Geometrical Standard Deviation (GSD). GSD is normally set equal to $1.5,2$ or 2.5 depending upon the concentration variability, i.e. (i) not very variable, (ii) variable and (iii) extremely variable.

Here after we report a sample log-normal table (Table 6) which includes a standard set of values for GM and GSD. As far as the entries are concerned, GSD can be chosen as the value corresponding to the most conservative case, i.e., normally 2,5, while $G M$ can be defined starting from $C_{c o r}$.

As mentioned before, $C_{c o r}$ can be assumed to be the geometric mean $G M$ or the simple mean $A M$. In the first case, its use is straightforward, in the second it's necessary to find a relation between the simple and the geometric mean of a lognormal distribution. Since a closed form equation does not exist and this relation also depends upon the values assumed by $G S D$, we must find a way to estimate $G M$ starting from $A M$. 
Table 6: $\quad$ Lognormal table.

\begin{tabular}{|c|c|c|c|}
\hline \multirow{2}{*}{ Probability } & \multicolumn{3}{|c|}{ GSD } \\
\cline { 2 - 4 } & 1,50 & 2,00 & 2,50 \\
\hline $0,10 \%$ & 0,010 & 0,002 & 0,000 \\
\hline $0,50 \%$ & 0,021 & 0,006 & 0,002 \\
\hline $1,00 \%$ & 0,031 & 0,010 & 0,003 \\
\hline $2,00 \%$ & 0,046 & 0,016 & 0,006 \\
\hline $3,00 \%$ & 0,060 & 0,023 & 0,009 \\
\hline $4,00 \%$ & 0,072 & 0,030 & 0,013 \\
\hline $5,00 \%$ & 0,085 & 0,037 & 0,016 \\
\hline $6,00 \%$ & 0,097 & 0,045 & 0,021 \\
\hline $7,00 \%$ & 0,109 & 0,052 & 0,025 \\
\hline $8,00 \%$ & 0,122 & 0,060 & 0,030 \\
\hline $9,00 \%$ & 0,134 & 0,068 & 0,035 \\
\hline $10,00 \%$ & 0,146 & 0,077 & 0,041 \\
\hline
\end{tabular}

A first consideration regards the fact that the relation among the geometrical mean and the simple mean is (Hardy, Littlewood, Pòlia - 1934):

$$
G M \leq A M
$$

i.e., the geometrical mean is always lower or equal to the simple mean or, written in a different way:

$$
A M=G M+\Delta
$$

Hereafter, we propose an experimental relationship between the two means for some specific values of GSD, i.e. the values $1.5,2$ and 2.5 mentioned before.

To study the relationship we created a calculation sheet in which for the three GSD values we generated, for fifteen values of geometrical mean, vectors of 100 randomized numbers distributed with the chosen value of $G S D$ and $G M$; for each vector, we computed the geometrical and simple mean. Using a 6-th grade polynomial interpolation function, it was possible to determine the relation between them. We used the 6-th grade after a set of experiments that revealed that the greater correlation factor was achieved using this grade of interpolation. Three functions were determined, one for each value of GSD. The following scheme, reported in Table 7, was used to generate the values:

Table 7: $\quad$ The experiment structure.

\begin{tabular}{|c|c|c|}
\hline \multicolumn{3}{|c|}{$\mathrm{GSD}_{\mathrm{i}}(\mathrm{i}=1, . ., 3)$} \\
\hline $\mathrm{GM}_{1}$ & $\ldots \ldots \ldots$ & $\mathrm{GM}_{15}$ \\
\hline $\log _{1}$ & $\ldots \ldots \ldots$ & $\log _{1}$ \\
\hline $\log _{2}$ & $\ldots \ldots \ldots$ & $\log _{2}$ \\
\hline$\vdots$ & $\ldots \ldots \ldots$ & $\vdots$ \\
\cline { 2 - 3 } & $\ldots \ldots \ldots$ & $\vdots$ \\
\hline $\log _{100}$ & $\ldots \ldots \ldots$ & $\log _{100}$ \\
\hline $\mathrm{GM}_{1}$ & $\ldots \ldots \ldots$ & $\mathrm{GM}_{15}$ \\
\hline $\mathrm{AM}_{1}$ & $\ldots \ldots \ldots$ & $\mathrm{AM}_{15}$ \\
\hline \multicolumn{3}{|c}{} \\
\hline
\end{tabular}


where $\log _{i}$ represents the $i$-th values of the vector generated from a log-normal distribution with parameters $G M j$ and GSDi.

The following figures 1,2 and 3 report the interpolation curves between simple mean AM and geometric mean GM found, respectively for GSD assuming the values $1.5,2$ and 2,5.

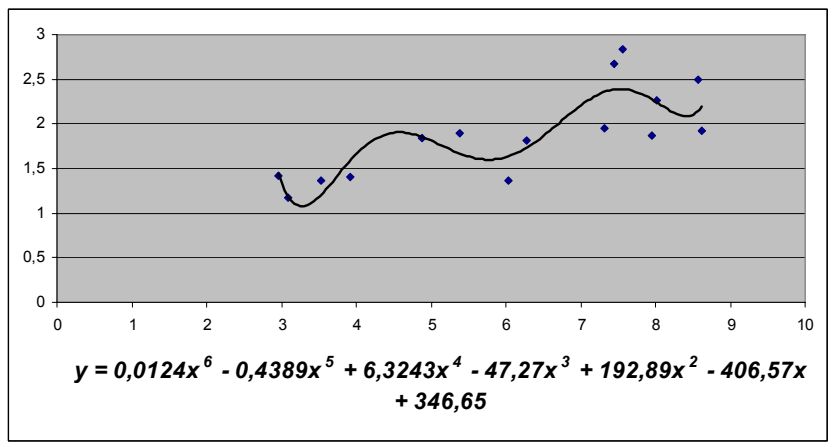

Figure 1: $\quad$ Interpolation between $\mathrm{AM}$ and GM - GSD = 1.5.

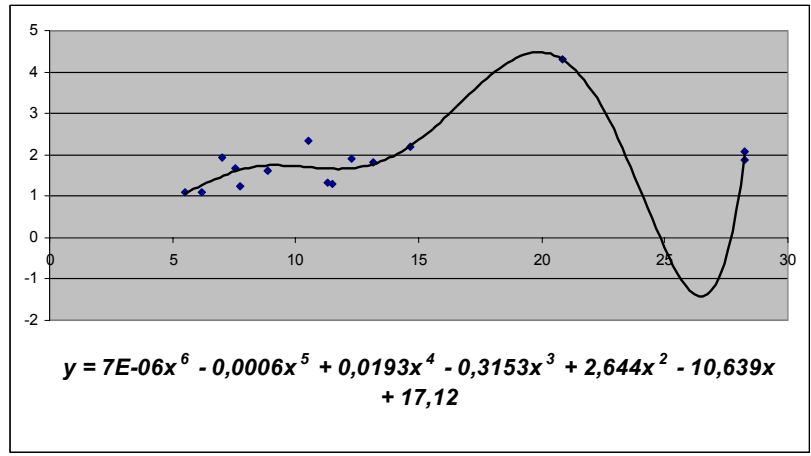

Figure 2: $\quad$ Interpolation between $\mathrm{AM}$ and $\mathrm{GM}-\mathrm{GSD}=2.0$.

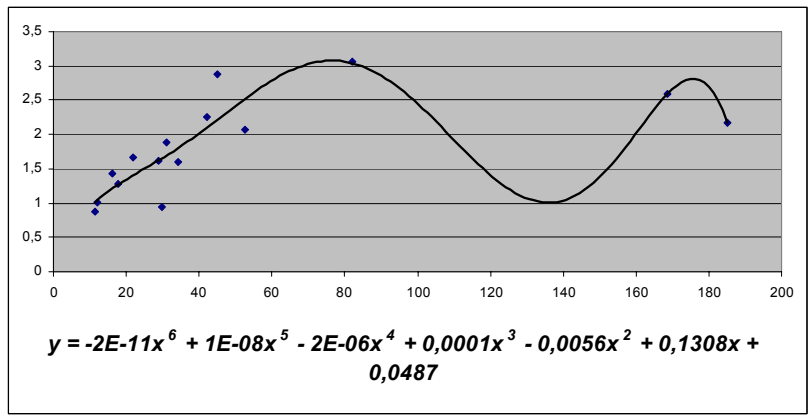

Figure 3: $\quad$ Interpolation between $\mathrm{AM}$ and GM - GSD = 2.5. 
The correlation coefficient for all the three relations is always over $77 \%$, which can be considered acceptable.

Thus, assuming $C_{c o r}$ is the simple mean, in order to compute the geometric mean of the distribution and, accordingly, enter the standard log-normal tables, it's simply necessary to apply the previous equation, one for each value of GSD.

After having shown the way to compute the geometrical mean, assuming that $C_{c o r}$ represents the simple mean, let us make some further consideration upon which is the most conservative choice to make: to consider $C_{c o r}$ to be either the geometric or the simple mean. In fact it is rather evident that making one choice or another leads to consider two completely different log-normal distribution curves for the chemical agent concentration.

As already mentioned, we know that, under the hypothesis of log-normal distribution, it is always verified that:

$$
G M \leq A M
$$

So, the two hypotheses are:

$$
\begin{aligned}
& \text { I) } C_{c o r}=G M \\
& \text { II) } C_{c o r}=A M=G M^{\prime}+\Delta
\end{aligned}
$$

Comparing the two relations, since $C_{c o r}$ is present in both ones, it holds that:

$$
G M=G M^{\prime}+\Delta \Rightarrow G M \geq G M^{\prime}
$$

So the geometrical mean $G M$ corresponding to the first choice is bigger than the geometrical mean $G M^{\prime}$ corresponding to the second one. This corresponds to the fact that the probability of exceeding a given TLV is greater when making the first choice, as it can be inferred by the previous Table 7 and the following Figure 4. Thus, in conclusion, the first choice is more conservative.

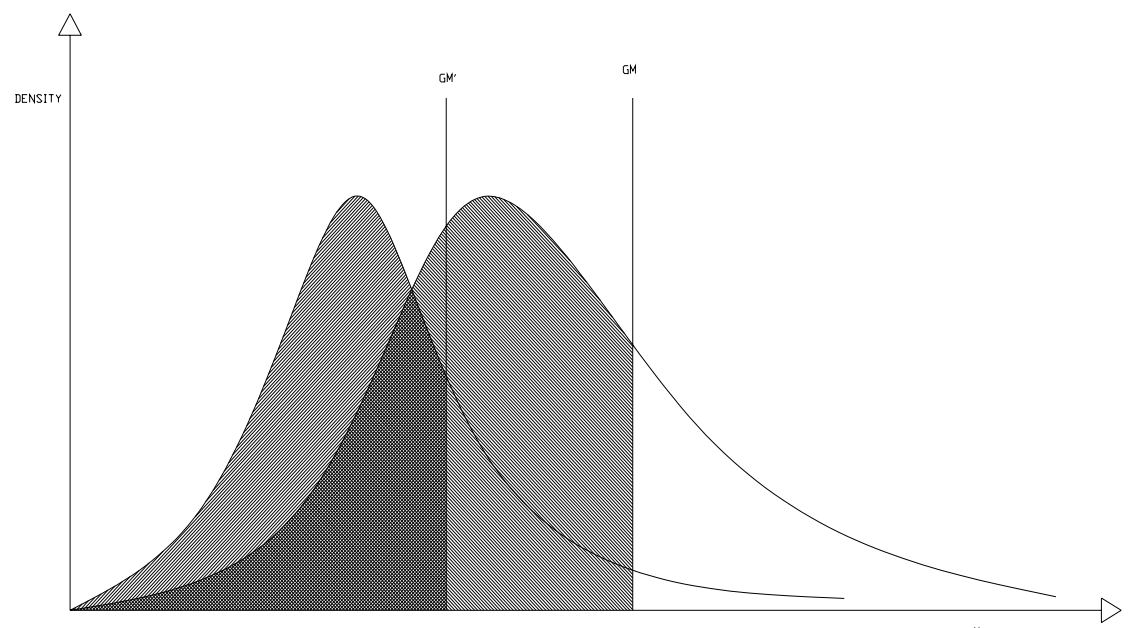

Figure 4: Log-normal distribution for the values of $G M$ and $G M^{\prime}$ 
Figure 4 reports two log-normal distributions with two different values of $G M$. The first curve, on the right, corresponds to a geometric mean $G M$ greater than the geometric mean GM' of the distribution of the left side.

Similarly, observing Figure 4, it can be easily inferred that, given a certain value of the concentration (reported on the x-axis), the area under the second curve (on the left side) is larger than the one under the first curve (on the right side).

\section{Conclusions}

In this work, after a study about the state of the art in chemical risk assessment, we noticed that the existing models are often difficult to use and require expensive experimental campaigns, and this is particularly true for SMEs.

So in this paper we proposed a new model for preliminary chemical risk assessment, which makes use of available data and, most importantly, is based on a simple calculation mechanism.

The aim of the model is to determine the probability that the concentration of dangerous chemical agents exceeds the TLV. When such probability goes over $10 \%$, this is an indication that further and deeper analysis is required. Thus, the proposed approach represents an improvement over the existing preliminary assessment model not only for the simplified mathematical approach just mentioned, but also because it reduces the set of chemical agents requiring deeper investigations, i.e. measurements campaigns.

Further improvements of this work include the development of automated calculation tools for a further simplification in the use of the assessment method.

\section{References}

[1] Scheringer et al. Scenario-Based Risk Assessment of Multi-Use Chemicals Application to Solvents. Risk Analysis, Vol.21, no. 3, 2001

[2] Ignatowsky and Rosenthal. The chemical accident risk assessment THESAURUS: A tool for analyzing and comparing diverse risk assessment processes and definitions. Risk Analysis, Vol.21, no.3, 2001

[3] Rood at al. Stochastic estimates of exposure and cancer risk from carbon tetrachloride released to the air from the Rocky Flats Plant - Risk Analysis, Vol.21, no.4, 2001

[4] Hwang and Chen. An evaluation of Risk Estimation Procedures for Mixtures of Carcinogens. Risk Analysis, Vol.19, no.6, 1999

[5] Demin V.F. Linear Dose-Effect relation for radiation and chemical carcinogenic risk. Atomic Energy, Vol.93, no. 4, 2002

[6] Roth C. Is Lognormal Kriging Suitable for local Estimation? Mathematical Geology, Vol.30, no.8, 1998

[7] Marcotte et Groleau. A simple and robust lognormal estimator. Mathematical Geology, Vol. 29, no.8, 1997

[8] Tarter and Hill. On thresholds and environmental curve tensiometers. Environmental and Ecological Statistics, no.4, 1997 
[9] Schulz and Griffin. Estimating Risk Assessment Exposure Point Concentration when the data are not normal or lognormal. Risk Analysis, Vol.19, no.4, 1999

[10] El-Baz and Nayak. Efficiency of composite sampling for estimating a lognormal distribution. Environmental and Ecological Statistics, no.11, 2004

[11] Dueholm et al. Assessment of exposure and health risk related to formaldehyde emissions from furniture: a case study. Indoor Air, no.5, 1995

[12] IEC 61508-5, Functional Safety of Electrical/Electronic/Programmable Electronic Safety Related Systems. Part 5 Examples of methods for determination of safety integrity levels, first edition 1998

[13] IEC 61508-6, Functional Safety of Electrical/Electronic/Programmable Electronic Safety Related Systems. Part 6 Guidelines on the application of IEC 61508-2 and IEC 61508-3, first edition, 2000

[14] EN 689, Guidance for the assessment of exposure by inhalation to chemical agents for comparison with limit values and measurements strategy, first edition, 1995

[15] De Cicco et al. Assessment strategy for chemical risk: critical analysis of the norms and standards. Ambiente e Sicurezza, no. 15, 2005

[16] Exposure, Version 2. A computer model for analyzing the effects of indoor air pollutant sources on individual exposures, Research Triangle Park, NC 27711, USA, US-EPA

[17] Gaylor and Chen: A simple upper limit for the sum of the risks of the components in a mixture, Risk Analysis, Vol. 14, n.6, 1996 